\title{
Relationship Between LDL-C Reduction After Coronary Revascularization and Prevention of Recurrent Cardiovascular Events
}

\author{
Tatsuhiro Nishiwaki ${ }^{1}$, Daisuke Kishi ${ }^{1}$, Fumihiko Yoshie $^{1}$, Keiko Fukuda ${ }^{1}$, Chikao Ibuki ${ }^{2}$, Yoshihiko Seino ${ }^{2}$, Mitsutoshi \\ Satoh $^{3}$ \\ ${ }^{1}$ Pharmaceutical Services Section, Nippon Medical School, Chiba Hokusoh Hospital, 1715 Kamagari, Inba-mura, \\ Inba-gun, Chiba, 270-1694, Japan. \\ ${ }^{2}$ Department of Internal Medicine, Nippon Medical School, Chiba Hokusoh Hospital, 1715 Kamagari, Inba-mura, \\ Inba-gun, Chiba, 270-1694, Japan. \\ ${ }^{3}$ Department of Toxicology and Pharmacology, Faculty of Pharmaceutical Sciences, Toho University, Miyama 2-2-1, \\ Funabashi, Chiba, 274-8510, Japan.
}

Received, November 23, 2009; Revised, January 26, 2010; Accepted, July 21, 2010; Published, July 22, 2010.

\begin{abstract}
Purpose: The present study was performed to determine whether improvement of the lipid profile during the first 3 months after coronary revascularization would allow prediction of the time until recurrence of cardiovascular events (CVEs). Methods. Blood biochemistry values of patients undergoing lipid-lowering therapy after undergoing coronary revascularization at Nippon Medical School Chiba Hokusoh Hospital, Japan, were investigated retrospectively. Recurrence of CVEs was defined as death, myocardial infarction, or angina requiring coronary revascularization 3 months or more after the first event. Results. Among 171 patients receiving secondary preventive care who suffered at least one recurrent CVE, 75 showed evidence of objective stenosis on coronary angiography. Among these 75 patients, exclusion of those for whom coronary revascularization had not been performed at disease onset, simple balloon angioplasty had been performed, serum lipid levels had not been measured, coronary revascularization had been applied for restenosis, or had not received statins left 37 patients suitable for inclusion in the study. Although the mean value of high-density-lipoprotein cholesterol did not change during the 3 months after the index coronary revascularization, that of low-density lipoprotein (LDL-C) decreased significantly. A significant positive correlation was identified between the percentage reduction in LDL-C during the first 3 months and the time until recurrence of CVEs $(\mathrm{r}=0.564, \mathrm{p}<0.01)$. The average LDL-C value $(106 \pm 24$ $\mathrm{mg} / \mathrm{dL})$ in patients who suffered CVE recurrence after 5 years tended to be lower than that $(125 \pm 38 \mathrm{mg} / \mathrm{dL})$ in patients who suffered recurrence within 5 years $(P=0.09)$. The incidence of patients achieving the LDL-C target level $(46.2 \%$ vs. $20.8 \% P<0.05)$ and the percentage reduction in LDL-C during the first 3 months $(-31.7 \pm 13.1 \%$ vs. $-12.3 \pm 18.9 \%, P<0.05)$ were significantly greater in patients who suffered CVE recurrence after 5 years than that in those who suffered recurrence within 5 years. Conclusion. The percentage reduction in LDL-C during the first 3 months after coronary revascularization, in addition to LDL-C values and achievement of the LDL-C goal, could serve as a useful predictor of CVE recurrence.
\end{abstract}

\section{INTRODUCTION}

Hypercholesterolemia is directly related to disorders of the endothelium and to the development or destabilization of atherosclerotic vascular disease [1-3]. Low-density-lipoprotein cholesterol (LDL-C) is a major factor in the progression of atherosclerotic lesions and atheroma destabilization [4-10]. The "Guideline for Diagnosis and Prevention of Atherosclerotic Cardiovascular Diseases in Japanese, 2007 Edition" [11], issued by the Japan Atherosclerosis Society, emphasizes the importance of managing not only total cholesterol (TC) but also LDL-C and high-density-lipoprotein cholesterol (HDL-C), and stratifies the risk of coronary artery disease (CAD) based on previous history of CAD and risk factors other than LDL-C. Patients with a history of CAD are classified as requiring secondary preventive care. The risk of CAD recurrence in patients receiving secondary preventive care is three times that of CAD development in patients receiving

Corresponding Author: Dr Mitsutoshi Satoh, Department of Toxicology and Pharmacology, Faculty of Pharmaceutical Sciences, Toho University, 2-2-1 Miyama, Funabashi, Chiba, 274-8510, Japan, E-mail: satoh@phar.toho-u.ac.jp 
primary preventive care $[12,13]$, and the guideline stipulates a lower LDL-C management goal (100 $\mathrm{mg} / \mathrm{dL}$ ) for patients requiring secondary prevention than for patients requiring primary prevention (Table 1).

3-Hydroxy-3-methylglutaryl coenzyme A reductase inhibitors (statins) are drugs commonly used to treat dyslipidemia. Many large-scale clinical trials have reported that treatment with statins lowers LDL-C levels in patients receiving secondary preventive care, and affords substantial prevention of CAD [14-16]. However, the extent to which a reduction in LDL-C during the early period after coronary revascularization can delay the onset of recurrent cardiovascular events (CVEs) has not yet been clarified.

In the present study, we investigated the relationship between the percentage change in LDL-C during the first 3 months after the index revascularization and the time until recurrence of CVEs. Our aim was to determine whether improvement of the lipid profile during the early period after coronary revascularization would allow prediction of the time until recurrence of CVEs.

\section{METHODS}

We performed this retrospective study at Nippon Medical School Chiba Hokusou Hospital, Chiba, Japan, after obtaining approval from the institutional ethics committee.

\section{Patient population}

We cross-sectionally surveyed 871 patients who visited Nippon Medical School Chiba Hokusou Hospital in the period between October and December 2007. The study targets were patients who had been diagnosed as having hyperlipidemia, hypercholesterolemia, or dyslipidemia and had been receiving a single statin drug for at least 3 months without a change in prescription, and whose serum levels of TC, LDL-C, HDL-C, and triglyceride (TG) had been evaluated appropriately during the same period.

Exclusion criteria included a diagnosis of familial hyper-cholesterolemia or secondary hyper cholesterolemia, development of myocardial infarction or angina within one month before the beginning of the target period, a diagnosis of chronic hepatitis or cirrhosis, use of hemodialysis, including peritoneal dialysis, and corticosteroid treatment. These exclusion criteria were factors that were considered to cause dyslipidemia but were not lifestyle-related. Moreover, patients taking more than one type of medication for their dyslipidemia were excluded.

There were 496 subjects with a medical history of myocardial infarction or angina (excluding vasospastic angina pectoris); these patients were considered to be receiving secondary preventive care. Among 171 of these patients who had suffered at least one episode of CVE recurrence, 75 showed objective evidence of angiographically determined coronary stenosis, and were therefore considered to have developed recurrent CAD.

Table 1. Lipid management goals based on risk assessment [11].

\begin{tabular}{|c|c|c|c|c|c|}
\hline \multirow[b]{2}{*}{ Principle of therapeutic strategy } & \multicolumn{2}{|c|}{ Category } & \multicolumn{3}{|c|}{ Lipid management goals $(\mathrm{mg} / \mathrm{dL})$} \\
\hline & & $\begin{array}{l}\text { Major risk factors } \\
\text { other than LDL-C* }\end{array}$ & LDL-C & HDL-C & TG \\
\hline Primary prevention & I (Low-risk group) & 0 & $<160$ & \multirow{5}{*}{$\geq 40$} & \multirow{5}{*}{$<150$} \\
\hline Lifestyle should be changed before & II (Intermediate-risk group) & $1 \sim 2$ & $<140$ & & \\
\hline consideration of drug therapy. & III (High-risk group) & 3 or more & $<120$ & & \\
\hline Secondary prevention & \multirow{2}{*}{\multicolumn{2}{|c|}{ History of coronary artery diseases }} & & & \\
\hline $\begin{array}{l}\text { Both drug therapy and lifestyle } \\
\text { modification are considered. }\end{array}$ & & & $<100$ & & \\
\hline
\end{tabular}

Management of serum lipids and intervention in other risk factors (smoking, hypertension, and diabetes) is necessary. *Major risk factors other than LDL-C are aging (male $>45$ years, female $>55$ years), hypertension, diabetes (including impaired glucose tolerance), smoking, family history of coronary artery disease, low HDL-C $(<40 \mathrm{mg} / \mathrm{dL})$. Category III, if complicated by diabetes mellitus, cerebral infarction or arteriosclerosis obliterans. 
After exclusion of patients who had not undergone coronary revascularization at the time of CAD onset, had undergone simple balloon angioplasty, had not undergone adequate measurement of serum lipid levels, in whom coronary revascularization had been applied to an area of restenosis, or statins untreated, 37 subjects suitable for evaluation remained. CVE recurrence was defined as death, myocardial infarction, or the need for coronary revascularization due to angina at least 3 months after the index coronary revascularization.

\section{Study protocols}

1. Patient characteristics

Data on age, gender, body height, body weight, risk factors for CAD except LDL-C, prescription drugs, and types of coronary revascularization were retrieved from the medical records.

\section{Biochemical test values}

Serum TC, LDL-C, TG, and HDL-C values were checked. If the LDL-C value had not been measured directly, it was calculated using the Friedewald equation [17]:

$$
\text { LDL-C }=\text { TC }- \text { HDL-C }- \text { TG } / 5(\mathrm{mg} / \mathrm{dL})
$$

However, if the TG value exceeded $400 \mathrm{mg} / \mathrm{dL}$, this equation was not applied, and the interval between the index coronary revascularization and the recurrence of CVEs was evaluated on the basis of medical records.

\section{Evaluation methods}

Percentage change in LDL-C was defined as the difference between the value measured at the time of index coronary revascularization and at 3 months (an average of $2.9 \pm 0.7$ months) after. The LDL-C management goal was defined as less than 100 $\mathrm{mg} / \mathrm{dL}$, in accordance with the Japan Atherosclerosis Society's 2007 guidelines mentioned above.

\section{Statistical analysis}

The data were analyzed statistically at $\mathrm{P}<0.05$ using the StatView ${ }^{\circledR}$ for Windows version 5.0 software package. Spearman's correlation rank test was applied to the correlation between the change in LDL-C and the time until recurrence. Although the unpaired t-test was employed to compare sample averages between patients with and without recurrence within 5 years, the Mann-Whitney U-test was employed when samples did not follow a normal distribution. The $\chi^{2}$ test was employed to compare any prevalences in the two groups. However, Fisher's exact probability test was employed when values were less than 5 .

\section{RESULTS}

\section{Patient characteristics}

The characteristics of the patients at the index coronary revascularization (baseline) are summarized in Table 2. Only two patients had no coronary risk factors at the time of index coronary revascularization. Acute myocardial infarction was the most prevalent diagnosis, and more than $60 \%$ of patients underwent percutaneous coronary intervention with a drug-eluting stent (DES). Among the statins, pravastatin was that most frequently prescribed. Seven patients received no statins.

\section{CVE recurrence}

The Kaplan-Meier curve for cumulative recurrence of CVEs is shown in Figure 1. The median time until recurrence was 3.1 years. Fourteen patients $(31.8 \%)$ were still recurrence-free at 5 years.

\section{Lipid profiles in the early period after index coronary revascularization}

Changes in lipid profiles during the early period after the index coronary revascularization are shown in Table 3. During this period, only TC and LDL-C were reduced significantly. Table 4 shows a comparison of TC, LDL-C, HDL-C and TG between patients in whom CVEs recurred within and after 5 years. A significant reduction of TC and LDL-C was observed in patients who suffered CVE recurrence after 5 years.

The level of LDL-C, the rate of achievement of the LDL-C management goal at 3 months after the index coronary revascularization, and the percentage reduction of LDL-C during the initial 3 -month period in patients with $(\mathrm{n}=24)$ and without $(n=13)$ CVE recurrence within 5 years are shown in Figure 2. The LDL-C level in patients without CVE recurrence within 5 years tended to be lower than that in patients with CVE recurrence within 5 years $(\mathrm{P}=0.09)$. The rate of achievement of the LDL-C target level $(46.2 \%$ vs. $20.8 \% \quad \mathrm{P}<0.05)$ and the percentage reduction of LDL-C were significantly greater in patients who remained CVE recurrence-free within 5 years than in patients who suffered recurrence during this period.

\section{Correlation between percentage reduction of $L D L-C$ and time until CVE recurrence}

Figure 3 shows a scattergram of the percentage reduction of LDL-C and period until CVE recurrence. There was a significant positive correlation between the two parameters $(\mathrm{r}=0.564$, $P<0.01)$. 
Table 2. Baseline characteristics of the patients.

\begin{tabular}{|c|c|c|}
\hline \multicolumn{2}{|c|}{$\begin{array}{c}\text { Sex (male no./female no.) } \\
\text { Age (year) } \\
\text { BMl }\left(\mathrm{kg} / \mathrm{m}^{2}\right)\end{array}$} & $\begin{array}{c}31 / 13 \\
62.7 \pm 10.5^{\star} \\
24.9 \pm 4.0^{\star}\end{array}$ \\
\hline & & \\
\hline \multirow{5}{*}{ Coronary risk factors } & Hypertension & $27(61.4)$ \\
\hline & Smoking & $24(54.5)$ \\
\hline & Family history & $17(38.6)$ \\
\hline & Diabetes & $24(54.5)$ \\
\hline & Chronic kidney disease & $16(36.4)$ \\
\hline \multirow{6}{*}{ Number of coronary risk factors } & 0 & $2(4.5)$ \\
\hline & 1 & $7(15.9)$ \\
\hline & 2 & $12(27.3)$ \\
\hline & 3 & $13(29.5)$ \\
\hline & 4 & $8(18.2)$ \\
\hline & 5 & $2(4.5)$ \\
\hline \multirow{4}{*}{ Diagnosis } & Acute myocardial infarction & $21(47.7)$ \\
\hline & Unstable angina & $6(13.6)$ \\
\hline & Effort angina & $14(31.8)$ \\
\hline & Others & $3(6.8)$ \\
\hline \multirow{3}{*}{ Coronary revascularization } & $\mathrm{PCl}$ with DES & $28(63.6)$ \\
\hline & $\mathrm{PCl}$ with BMS only & $13(29.5)$ \\
\hline & CABG & $3(6.8)$ \\
\hline \multirow{6}{*}{$\begin{array}{c}\text { Statins } \\
\text { or } \\
\text { other treatement }\end{array}$} & Pravastatin & $18(40.9)$ \\
\hline & Simvastatin & $5(11.4)$ \\
\hline & Fluvastatin & $5(11.4)$ \\
\hline & Atorvastatin & $7(15.9)$ \\
\hline & Pitavastatin & $2(4.5)$ \\
\hline & Exercise and diet & $7(15.9)$ \\
\hline
\end{tabular}

DES, drug-eluting stent; BMS, bare metal stent; CABG, coronary artery bypass grafting. *mean \pm S.D.

\section{DISCUSSION}

The present study clearly indicated a significant correlation between percentage reduction of the LDL-C level during the early period (3 months) after the index coronary revascularization and the time until CVE recurrence, suggesting that LDL-C reduction in the early period after index coronary revascularization could affect the period until CVE recurrence.

When we divided patients into two groups according to whether CVEs recurred within and beyond 5 years, the former showed a high LDL-C level and a low rate of achieving the LDL-C management goal in the first 3 months after index revascularization. These findings support the 2007 Guideline of the Japan Atherosclerosis Society, which considers achievement of the LDL-C management goal (LDL-C $<100 \mathrm{mg} / \mathrm{dL}$ ) important for patients receiving secondary preventive care [11, 18]. However, the average LDL-C value 3 months after the index coronary revascularization in patients who suffered CVE recurrence after 5 years was over $100 \mathrm{mg} / \mathrm{dL}(103 \pm 22 \mathrm{mg} / \mathrm{dL})$. This implies that CVE recurrence can be delayed with sufficient reduction of LDL-C during the early period after index coronary revascularization, even if LDL-C still exceeds the management goal. Thus, the rate of LDL-C reduction might have a substantial impact in preventing or delaying the recurrence of CVEs that is as potent as the LDL-C level or the rate of achieving the LDL-C management goal. The J-LIT, which targeted patients with low-dose simvastatin therapy in secondary preventive care, reported that $10 \mathrm{mg} / \mathrm{dL}$ decrease in LDL-C and $10 \mathrm{mg} / \mathrm{dL}$ increase in HDL-C concentration reduce the risk of CHD by $8.0 \%$ and $28.3 \%$ respectively [13]. Although we did not survey the cumulative relapse ratio of the patients without statin treatment in our study, the Kaplan-Meier curve of cumulative relapse rations of the patients might be drawn below that of the patients with statin treatment. 


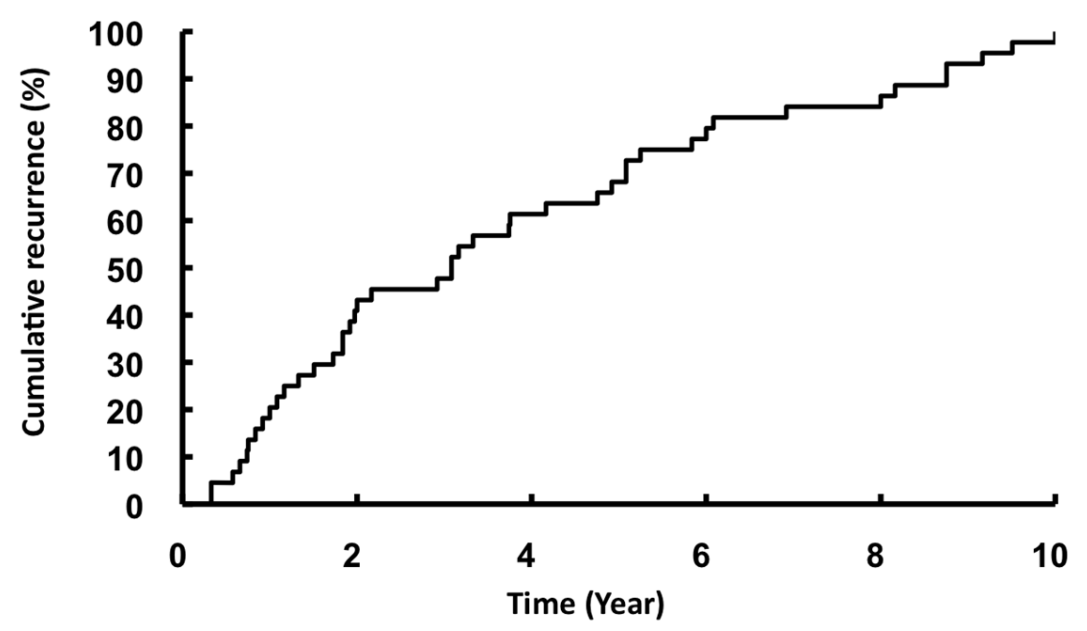

Figure 1. Kaplan-Meier curve showing cumulative recurrence of CVE.

\begin{tabular}{|c|c|c|c|c|}
\hline & baseline & after 3 months & $\%$ change & $* p$ value \\
\hline $\mathrm{TC}$ & $226 \pm 43$ & $198 \pm 37$ & $-9.4 \pm 22.7$ & $<0.01$ \\
\hline LDL-C & $148 \pm 26$ & $118 \pm 31$ & $-19.1 \pm 19.3$ & $<0.01$ \\
\hline HDL-C & $49 \pm 13$ & $51 \pm 13$ & $9.5 \pm 27.3$ & N.S. \\
\hline TG & $152 \pm 58$ & $137 \pm 52$ & $-4.3 \pm 34.4$ & N.S. \\
\hline
\end{tabular}

TC: total cholesterol, HDL-C: high-density-lipoprotein cholesterol, TG: triglyceride. $\mathrm{n}=37$ ${ }^{*} p$ value: baseline vs. after 3 months by paired t-test

Lipid-lowering therapy plays an important role in preventing the recurrence of CVE regardless of whether a patient is receiving primary or secondary prevention. On the basis of recent guidelines, the LDL-C management goal is stratified according to the individual risk in each patient [11, 19]. In patients receiving secondary prevention, like those in the present study, the risk of CVE recurrence is estimated to be 3-4 times greater than in patients receiving primary prevention $[12,13]$. In any guideline, the lowest LDL-C value is allocated as a management goal for patients receiving secondary prevention. However, the period during which the LDL-C management goal should be achieved, and how soon the LDL-C level should be lowered, are not stipulated. It has been reported that in patients with acute coronary syndrome (ACS), recurrence of
CVEs within 16 weeks after onset of ACS can be suppressed if administration of atorvastatin is started within 24-96 hours of presentation [20]. Furthermore, it has been proposed that statin treatment in the early phase of ACS may improve the long-term outcome [21, 22]. However, no previous report has evaluated the association between improvement of the lipid profile in the early period after index coronary revascularization and the period until CVE recurrence. The results of the present study suggest that improvement of the lipid values during the first 3 months after index coronary revascularization could be a useful predictor of the period until CVE recurrence.

Studies using IVUS and/or angioscopy have revealed multiple vulnerable plaques in the coronary arteries of patients with ACS or stable angina [23-25]. 
Table 4. Efficacy of statin treatment for 3 months on TC, LDL-C, HDL-C and TG in patients classified according to CVE recurrence within and after 5 years.

\begin{tabular}{lcccccc}
\hline & CVE recurrence & baseline & after 3 months & $* p$ value & $\%$ change & $* * p$ value \\
\hline \multirow{2}{*}{ TC } & within 5 years & $225 \pm 37$ & $214 \pm 54$ & N.S. & $-5.0 \pm 17.3$ & $<0.05$ \\
& after 5 years & $230 \pm 55$ & $186 \pm 31$ & $<0.01$ & $-13.4 \pm 30.4$ & \\
\multirow{2}{*}{ LDL-C } & within 5 years & $147 \pm 27$ & $135 \pm 46$ & N.S. & $-8.7 \pm 21.3$ & $<0.01$ \\
& after 5 years & $151 \pm 26$ & $103 \pm 23$ & $<0.01$ & $-31.0 \pm 11.4$ & \\
\multirow{2}{*}{ HDL-C } & within 5 years & $49 \pm 12$ & $52 \pm 12$ & N.S. & $7.6 \pm 23.9$ & N.S. \\
& after 5 years & $49 \pm 14$ & $50 \pm 13$ & N.S. & $8.6 \pm 31.3$ & \\
\multirow{2}{*}{ TG } & within 5 years & $149 \pm 52$ & $134 \pm 57$ & N.S. & $-7.3 \pm 29.7$ & N.S. \\
& after 5 years & $159 \pm 65$ & $144 \pm 56$ & N.S. & $-2.1 \pm 44.4$ & \\
\hline
\end{tabular}

TC: total cholesterol, HDL-C: high-density-lipoprotein cholesterol, TG: triglyceride. $\mathrm{n}=37$

${ }^{*} p$ value: baseline vs. after 3 months by paired t-test. ${ }^{*} p$ value: CVE recurrence within 5 years vs. after 5 years by Mann-Whitney $U$ test

As these vulnerable plaques might ultimately rupture, resulting in recurrence of CVEs [26], aggressive medical therapy to stabilize them should be introdued after coronary revascularization. In this connection, statins exert various pleiotropic actions to reduce the vulnerability of plaque, independent from their lipid-lowering activity, including improvement of endothelial function [27-29], and anti-thrombotic [30, 31] and anti-inflammatory $[32,33]$ activities. The implication of the present findings, that a greater reduction in the LDL-C level during the early period after index coronary revascularization could delay the recurrence of CVEs, may reflect the above pathological process. Because the design of the present study excluded any restenosis-related events that could have led to CVE recurrence, the administration of statin may have stabilized any non-culprit plaque that had disseminated in the coronary artery.

The present study had several limitations. First, it was a retrospective study, and therefore clinical information that could not be evaluated (e.g. death) was not considered, as we focused on patients with previous coronary revascularization who visited our hospital outpatient department during the cross-sectional survey period. It will therefore be necessary to perform a prospective study to examine the relationship between the percentage reduction in LDL-C and the development of CVEs, including death, in patients undergoing coronary revascularization. Second, the sample size was small because only patients with recurrence of CVEs identified as CAD were included. However, it was prerequisite to ensure the reliability of each event by coronary angiography, because the present study focused chiefly on the period until CVE recurrence. Finally, the study did not include patients free from CVEs, i.e. we were unable to determine how the lipid-lowering effect contributed to reduction of the CVE recurrence rate. As mentioned above, our study aim was to investigate the relationship between the early reduction of the LDL-C level after index coronary revascularization and the period until CVE recurrence. Therefore, investigation of the incidence of CVEs was beyond the scope of the study.

In conclusion, the present study has demonstrated a positive correlation between reduction of the LDL-C level during the first 3 months after index coronary revascularization and the recurrence of CVEs, suggesting that aggressive lipid-lowering therapy, particularly in the early phase, could delay CVE recurrence. The rate of LDL-C reduction in the early phase could be as useful a marker for prediction of CVE recurrence as the LDL-C level per se or the rate of achieving the LDL-C management goal.

\section{Acknowledgment}

This research was supported by a Special Grant to members of the Faculty of Pharmaceutical Sciences, Toho University. 
A

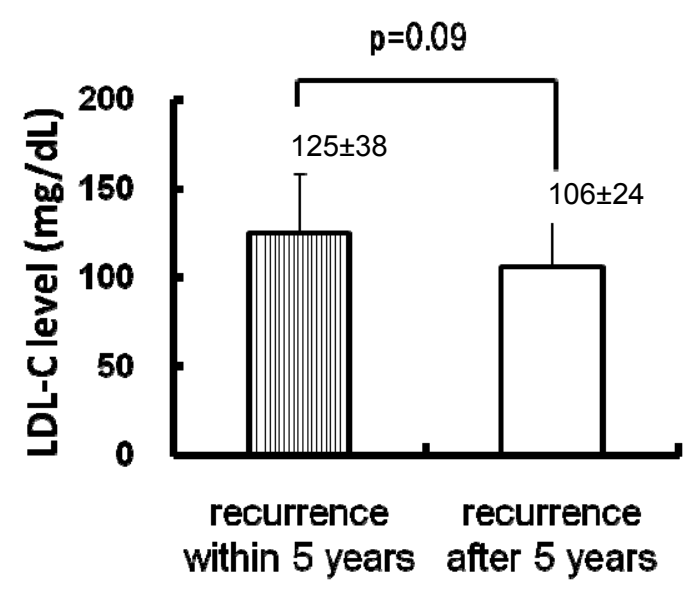

B

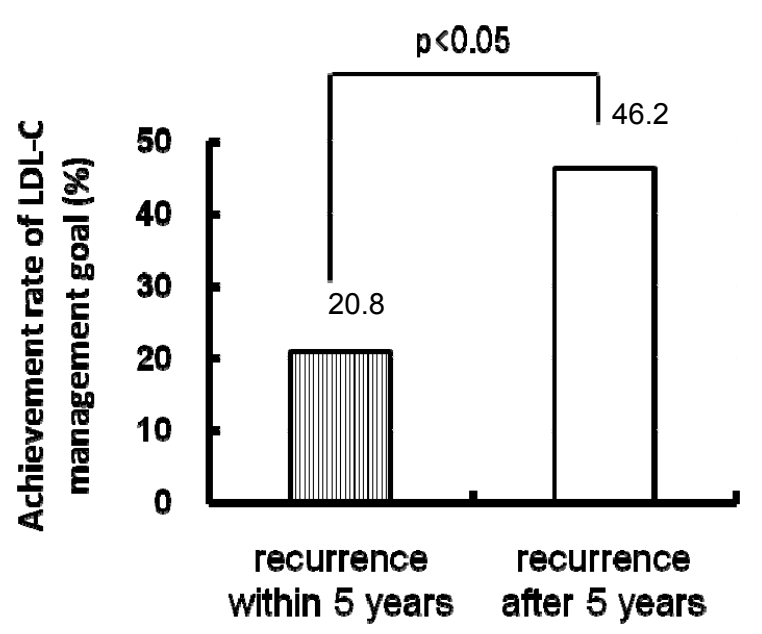

C

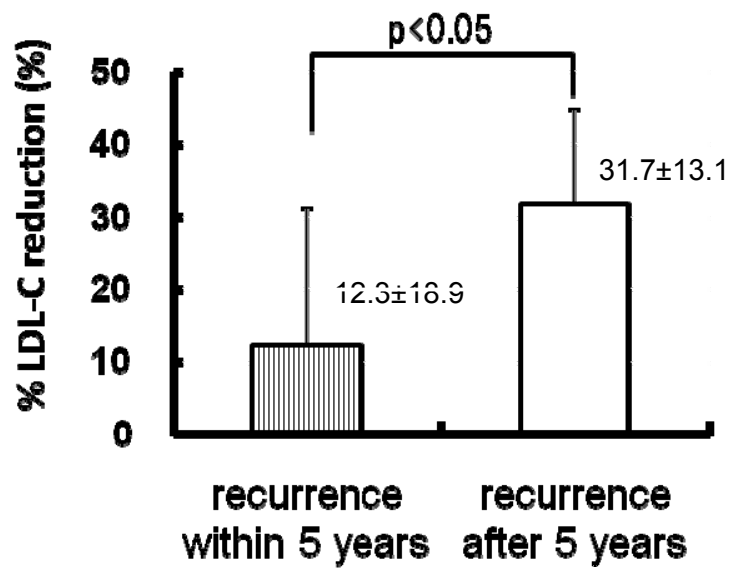

Figure 2. LDL-C level (A), rate of achievement of the LDL-C management goal (B), and percentage reduction of LDL-C (C) in patients who suffered CAD recurrence within 5 years $(n=24)$ and after 5 years $(n=13)$. 


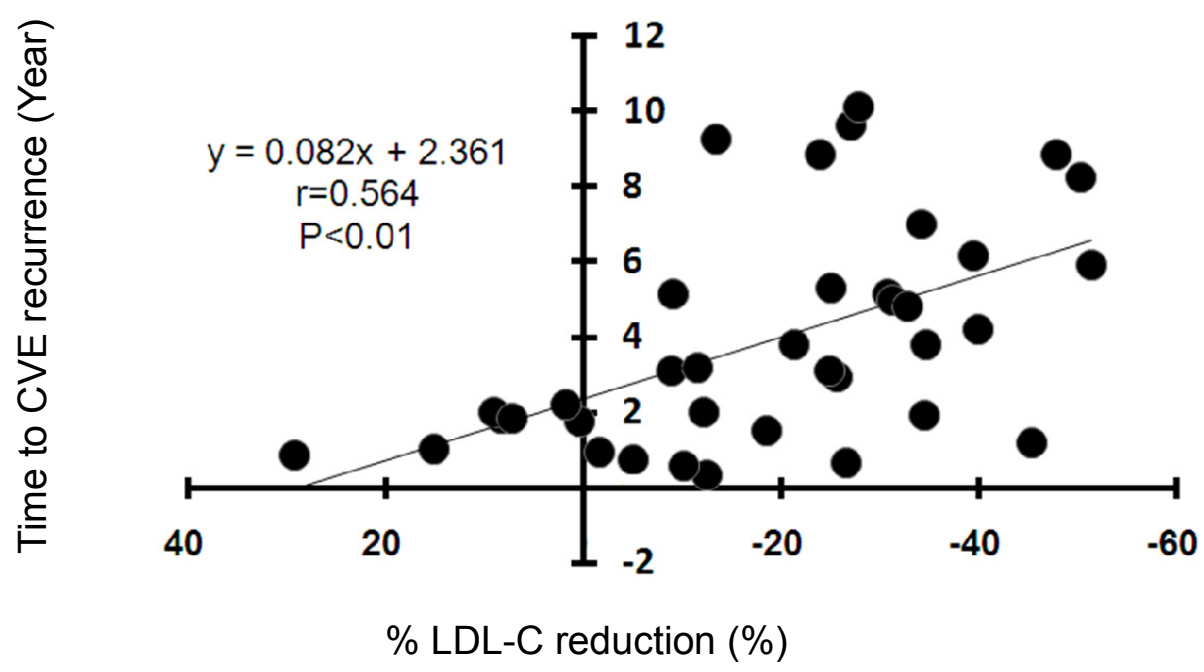

Figure 3. Correlation between percentage reduction of LDL-C and period until CVE recurrence $(n=37)$.

\section{REFERENCES}

1. Ross R, Harker L: Hyperlipidemia and atherosclerosis. Science 193: 1094-1100, 1976.

2. Ross R: Atherosclerosis-an inflammatory disease. New Engl J Med 340: 115-126, 1999.

3. Ross R: The pathogenesis of atherosclerosis: a perspective for the 1990s. Nature 362: 801-809, 1993.

4. Carleton RA, Dwyer J, Finberg L, Flora J, Goodman DS, Grundy SM, Havas S, Hunter GT, Kitchevsky D, Lauer RM: MRFIT research group: Report of the Expert Panel on Population Strategies for Blood Cholesterol Reduction. A statement from the National Cholesterol Education Program, National Heart, Lung, and Blood Institute, National Institutes of Health. Circulation 83: 2154-2232, 1991.

5. Nakamura Y, Yamamoto T, Okamura T, Kadowaki T, Hayakawa T, Kita Y, Saitoh S, Okayama A, Ueshima H: The NIPPON DATA 80 research group: Combined cardiovascular risk factors and outcome NIPPON DATA 80, 1980-1994. Circ J 70: 960-964, 2006.

6. Okamura T, Tanaka H, Miyamatsu N, Hayakawa T, Kadowaki T, Kita Y, Nakamura Y, Okayama A, Ueshima H: The NIPPON DATA80 research group : The relationship between serum total cholesterol and all-cause or cause-specific mortality in a 17.3-year study of a Japanese cohort. Atherosclerosis 190: 216-223, 2007.

7. Okamura T, Kadowaki K, Hayakawa T: The NIPPON DATA80 research group: What cause of mortality can we predict by cholesterol screening in the Japanese general population? Int J Med 253: 169-180, 2003.

8. Matsuzaki M, Kita T, Mabuchi H, Matsuzawa Y,
Nakaya N, Oikawa S, Saito Y, Sasaki J, Shimamoto K, Itakura H: J-LIT Study Group: Large scale cohort study of the relationship between serum cholesterol concentration and coronary events with low-dose simvastatin therapy in Japanese patients with hypercholesterolemia. Circ J 66: 1087-1095, 2002.

9. Matsuzawa Y, Kita T, Mabuchi H, Matsuzaki M, Nakaya N, Oikawa S, Saito Y, Sasaki J, Shimamoto K, itakura H: J-LIT Study Group: Sustained reduction of serum cholesterol in low-dose 6-year simvastatin treatment with minimum side effects in 51,312 Japanese hypercholesterolemic patients. Circ J 67: 287-294, 2003.

10. Horiuchi H, Kita T, Mabuchi H, Matsuzawa Y, Nakaya N, Oikawa S, Saito Y, Sasaki J, Shimamoto $\mathrm{K}$, Itakura H: J-LIT Study Group : Primary cardiovascular events and serum lipid levels in elderly Japanese with hypercholesterolemia undergoing 6-year simvastatin treatment : A subanalysis of the Japan Lipid Intervention Trial. $J$ Am Geriatr Soc 52: 1981-1987, 2004.

11. Japan Atherosclerosis Society: "The guideline for diagnosis andprevention of atherosclerotic cardiovascular diseases for Japanese 2007 edition".

12. O'Keefe JH Jr, Cordain L, Harris WH, Moe RM, Vogel R: Optimal low-density lipoprotein is 50 to 70 $\mathrm{mg} / \mathrm{dl}$ : lower is better and physiologically normal. $J$ Am Coll Cardiol 43: 2142-2146, 2004.

13. Mabuchi H, Kita T, Matsuzaki M, Matsuzawa Y, Nakaya N, Oikawa S, Saito Y, Sasaki J, Shimamoto $\mathrm{K}$, Itakura H: J-LIT study group: Large scale cohort study of the relationship between serum cholesterol concentration and coronary events with low-dose simvastatin therapy in Japanese patients with hypercholesterolemia and coronary heart disease-secondary prevention cohort study of the 
Japan Lipid Intervention Trial (J-LIT): Circ $J$ 66: 1096-1100, 2002.

14. Scandinavian simvastatin survival study group: Randomized trial of cholesterol lowering in 4444 patient with coronary heart disease: The scandinavia simvastatin survival study (4S). Lancet 344: 1383-1389, 1994.

15. Sacks FM, Pfeffer MA, Moye LA, Rouleau JL, Rutherford JD, Cole TG, Brown L, Warnica JW, Arnold JMO, Wun CC, Davis BR, Braunwald E: The cholesterol and, for recurrent enents trial investigators: The effect of pravastatin on coronary events after myocardial infarction in patients with average cholesterol levels. New Engl J Med 335: 1001-1009, 1996.

16. The long-term intervention with pravastatin in ischemic disease (LIPID) study group: Prevention of cardiovascular events and death with pravastatin in patients with coronary heart disease and a broad range of initial cholesterol levels. $N$ Engl J Med 339: 1349-1357, 1998.

17. Friedewald WT, Levy RI, Fredrickson DS: Estimation of the concentration of low-density lipoprotein cholesterol in plasma, without use of the preparative ultracentrifuge. Clin Chem 18: 499-502, 1972.

18. Ballantyne CM: Low-density lipoproteins and risk for coronary artery disease. Am $J$ Cardiol 82: 3Q-12Q, 1998.

19. Implications of recent clinical trials for the national cholesterol education program adult treatment panel III guidelines. Circ 110: 227-239, 2004.

20. Schwartz GG, Olsson AG, Ezekowitz MD, Ganz P, Oliver MF, Waters D, Zeiher A, Chaitman BR, Leslie S, Stern T: Myocardial ischemia reduction with aggressive cholesterol lowering (MIRACL) study investigators. Effects of atorvastatin on early recurrent ischemic eventsin acute coronary syndromes The MIRACL study: A sandomized controlled trial. JAMA.285: 1711-1718, 2001.

21. Thompson PL: Clinical relevance of statins: instituting treatment early in acute coronary syndrome patients. Atheroscler suppl 2:15-9, 2001.

22. Thompson PL, Meredith I, Amerena J, Campbell TJ, Sloman JG, Harris PJ: Effect of pravastatin compared with placebo initiated within 24 hours of onset of acute myocardial infarction or unstable angina: The pravastatin in acute coronary treatment (PACT) trial. Am Heart J 148: e2, 2004.

23. Rioufol G, Finet G, Ginon I, André-Fouët X, Rossi R, Vialle E, Desjoyaux E, Convert G, Huret JF, Tabib A: Multiple atherosclerotic plaque rupture in acute coronary syndrome: a three-vessel intravascular ultrasound study. Circ 106: 804-808, 2002.
24. Hong MK, Mintz GS, Lee CW, Kim YH, Lee SW, Song JM, Han KH, Kang DH, Song JK, Kim JJ, Park SW, Park SJ: Comparison of coronary plaque rupture between stable angina and acute myocardial infarction: a three-vessel intravascular ultrasound study in 235 patients. Circ 110: 928-33, 2004.

25. Asakura M, Ueda Y, Yamaguchi O, Adachi T, Hirayama A, Hori M, Kodama K: Extensive development of vulnerable plaques as a pan-coronary process in patients with myocardial infarction: an angioscopic study. $\mathrm{J} \mathrm{Am}$ Coll Cardiol 37: 1284-1288, 2001

26. Libby P: Current concepts of the pathogenesis of the acute coronary syndromes. Circ 104: 365-372, 2001.

27. O'Driscoll G, Green D, Taylor RR: Simvastatin, an HMG-coenzyme A reductase inhibitor, improves endothelial function within 1 month. Circ 95: 1126-1131, 1997.

28. Huggins GS, Pasternak RC, Alpert NM, Fischman AJ, Gewirtz H: Effects of short-term treatment of hyperlipidemia on coronary vasodilator function and myocardial perfusion in regions having substantial impairmentof baseline dilator reserve. Circ 98: 1291-1296, 1998.

29. Dupuis J, Tardif J-C, Cernacek P, The' roux P: Cholesterol reduction rapidly improves endothelial function after acute coronary syndromes: the RECIFE (Reduction of cholesterol in ischemia and function of endothelium) Trial. Circ 99: 3227-3333, 1999.

30. Lacoste L, Lam JYT, Hung J, Letchacovski G, Solymoss CB, Waters D: Hyperlipidemia and coronary disease: correction of the increased thrombogenic potential with cholesterol reduction. Circ 92: 3172-3177, 1995.

31. Notarbartolo A, Davì G, Averna M, Barbagallo CM, Ganci A, Giammarresi C, La Placa FP, Patrono C: Inhibition of thromboxane biosynthesis and platelet function by simvastatin in type IIa hypercholesterolemia. Arterioscler Thromb Vasc Bio. 15: 247-251, 1995.

32. Bustos C, Hernández-Presa MA, Ortego M, Tuñón J, Ortega L, Pérez F, Díaz C, Hernández G, Egido J: HMG-CoA reductase inhibition by atorvastatin reduces neointimal inflammation in a rabbit model of atherosclerosis. J Am Coll Cardiol 32: 2057-2064, 1998.

33. Ridker PM, Rifai N, Pfeffer MA, Sacks F, Braunwald E: Long-term effects of pravastatin on plasma concentration of C-reactive protein. Circ 100: 230-235, 1999. 